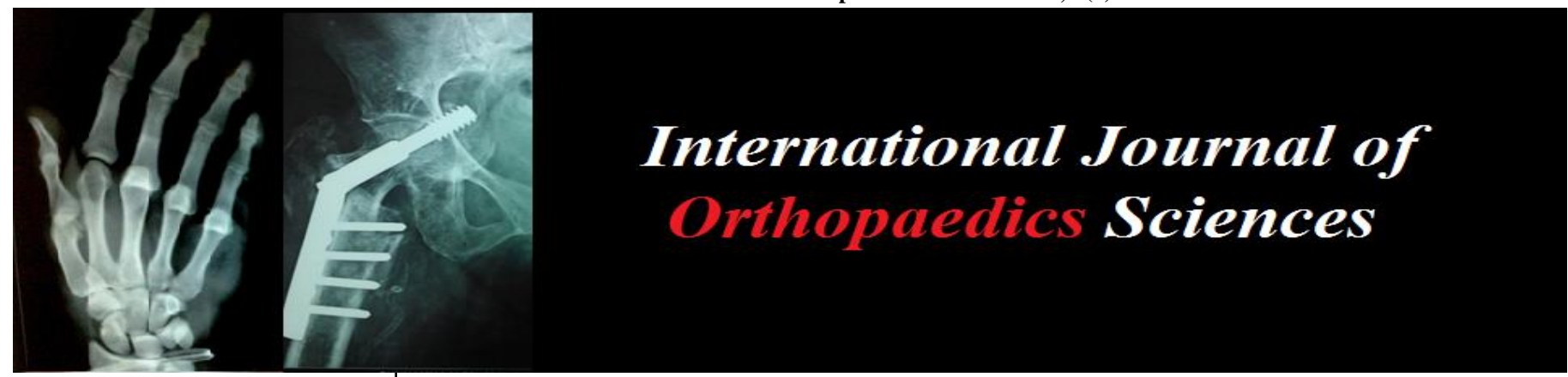

ISSN: $2395-1958$

IJOS 2018; 4(2): 812-815

(C) 2018 IJOS

www.orthopaper.com

Received: 27-02-2018

Accepted: 28-03-2018

Naveen PR

Associate Professor,

Dept of Orthopaedics, Shimoga

Institute of Medical Sciences,

Shimoga, Karnataka, India

Kiran Kumar HG

Assistant Professor, Dept of Orthopaedics, Shimoga Institute of Medical Sciences, Shimoga

Chaitanya PR

Professor, Dept of Orthopaedics, Shimoga Institute of Medical

Sciences, Shimoga, Karnataka, India

Ramachandra N Badami

Dept of Orthopaedics, Shimoga

Institute of Medical Sciences,

Shimoga, Karnataka, India
Correspondence

Kiran Kumar HG

Assistant Professor, Dept of

Orthopaedics, Shimoga Institute

of Medical Sciences, Shimoga,

Karnataka, India

\section{Prospective randomised control study of role of proximal femur nail and dynamic hip screw and plate in unstable intertrochanteric fractures of the femur in elderly}

\author{
Naveen PR, Kiran Kumar HG, Chaitanya PR and Ramachandra N \\ Badami
}

DOI: https://doi.org/10.22271/ortho.2018.v4.i21.117

\section{Abstract}

Introduction: The conservative treatment of intertrochanteric (IT) fracture has high complication rate. The operative management of intertrochanteric fractures are sucussful. Commonly used implants for fixation of IT fractures are dynamic hip screw (DHS) and proximal femur nail (PFN). Debate still continues regarding the optimal implant for IT fractures. We aim to compare the results of DHS and PFN in the treatment of IT fractures at our institution.

Materials and Methods: This is a prospective randomized comparative study of 40 patients of IT fractures treated surgically. Patients were divided equally into 2 groups of 20 each, with patients in group-A treated with DHS and those in group-B PFN. The functional outcome was evaluated based on Harris Hip Score (HHS). The radiographic outcomes was based on non-union or mal-union, femoral head necrosis, cut-out, periimplant fracture.

Results: The Harris Hip Score in the D.H.S group at 1 month (Avg. 24.4) was less than that of the P.F.N group (Avg. 33), $p<0.05$. During follow-up period, Group PFNA improved from $33 \pm 2.99$ to $87.62 \pm$ 7.53, and Group DHS improved from $24.4 \pm 2.98$ to $74.44 \pm 7.95$. Patients in PFN group had fewer complications as compared to those in DHS group and the difference was statistically significant.

DISCUSSION: the most important result is that patients treated with the PFN technique exhibited functional improvement as early as 6 months after surgery, unlike DHS-treated patients. In practice, this means that PFN treatment is associated with faster improvement in quality of life than the DHS technique.

Conclusion: PFN is better than DHS, as indicated by significantly less total complications, less reoperation rate, and higher postoperative HHS

Keywords: Intertrochanteric, fracture, DHS, PFN

\section{Introduction}

Hip fracture is a severe and common injury that occurs predominantly in the elderly ${ }^{[1-3]}$. It is the leading cause of morbidity and mortality in this population ${ }^{[4-6]}$. The incidence of intertrochanteric (IT) fractures of the femur is race- and sex dependent, and varies across countries. These fractures have a long-term impact on the quality of life of patients and their carers. The functional outcomes of patients with intertrochanteric fractures can be surprisingly poor, and many become limited to ambulation within the home and dependent on carers for the activities of daily living ${ }^{[7]}$. The various treatment options for intertrochanteric fractures are operative and nonoperative.

The conservative approach has high complication rate. The common problems of prolonged immobilization, decubitus ulcers, urinary tract infection, joint contractures, pneumonia, and thromboembolism contribute to the high mortality rate. The increased incidence of varus deformity and shortening results in poor function. The operative management of intertrochanteric fractures has evolved since usage of fixed nail plate, dynamic hip screws to which several modifications have been added to intramedullary devices. 
The dynamic hip screw (DHS) fixation device is widely used for the surgical treatment of intertrochanteric fractures of the femur ${ }^{[8,9]}$. This device is considered to be the gold standard for management of these fractures in the elderly ${ }^{[10]}$. In 1996, $\mathrm{AO} / \mathrm{ASIF}$ developed the proximal femoral nail (PFN) as an intramedullary fixation device for pertrochanteric, intertrochanteric, and subtrochanteric fractures of the femur [11]. The PFN is a minimally-invasive implant and a good treatment option for fractures of the proximal end of the femur, particularly when closed reduction is possible ${ }^{[11]}$.

But there is a debate as which implant should be used in unstable IT fractures with special mention to osteoporotic bone and old age. Our study was aimed at comparing the dynamic hip screw (DHS) with the Proximal femur nail (PFN) in the surgical management of unstable intertrochanteric fractures prospectively.

\section{Materials and methods}

This is a randomized control trial conducted between June 2016 to June 2017 at the Govt McGann hospital, SIMS, Shivamogga. After obtaining ethical clearance from the institutional ethical committee, 40 cases with post traumatic unstable IT fracture of femur in elderly patients who were treated surgically either with DHS or PFN were included for the study. Consent was taken from all participants of the study. Exclusion criteria were: pathological fractures or the presence of metastatic disease, poly-trauma, severe osteoarthritis, chemotherapy, ASA score V, patients with other associated injuries.

The patients were divided into two demographically identical groups of 20 patients each. The patients were randomly divided into the 2 groups based on the computer generated random numbers. The patients in Group-A were surgically treated with Dynamic hip screw (DHS) and the patients in Group-B were surgically treated with a Proximal femur nail (PFN).

Patients were operated on as soon as any necessary optimisation of existing medical comorbidities was completed. Prior to fixation, all the fractures were reduced on a fracture table under fluoroscopic guidance, and prophylactic antibiotics were given. In patients having PFN fixation, weight bearing ambulation and rehabilitation was commenced on day 2. The extent of weight bearing was decided by the operating surgeon based on the stability of the fracture. In patients having DHS fixation, non-weight bearing ambulation with walking aid was commenced on day 2 .

The intra- and post-operative outcomes included osteosynthesis complications, and postoperative Harris Hip Score (HHS). The radiographic outcomes (non-union or malunion, femur shaft fracture after implant removed, femoral head necrosis, cut-out, periimplant fractures) was assessed immediately at 1, 2, 3, 6, 9, 12 months postoperatively or at the final follow-up. The mean follow-up was 10 months and a maximum follow-up was 1 year and a minimum follow-up was 9 months.

Mal-union was defined as less than $50 \%$ contact between the proximal and distal fragments or collodiaphyseal angle (CCD) of less than $120^{\circ}$. Non-union was defined as lack of union after six months of follow-up. Deep wound infection and superficial wound infection was respectively defined as an established infection beneath the fascia requiring surgical revision and a cutaneous or subcutaneous infection requiring antibiotic therapy. Implant failure was defined as any condition which would necessitate revision surgery with change of implant. All other complications were noted.

\section{Results}

The study included 40 elderly patients of IT fracture of femur divided into two groups. Both the groups were equal, identical and demographically matched. The overall mean age was 76.8 (range 66-85) years with $56 \%$ of the patients being female and $44 \%$ males. $86 \%$ of the patients were either independent community ambulatory or community ambulant and the rest $14 \%$ of the patients were home ambulant or used assistive devices for ambulation prior to the IT fracture of femur. mean follow-up period was 53 months (range, 48-60 months).

The Harris Hip Score in the D.H.S group at 1 month (Avg. 24.4) was less than that of the P.F.N group (Avg. 33), $p<0.05$. During follow-up period, Group PFNA improved from $33 \pm$ 2.99 to $87.62 \pm 7.53$, and Group DHS improved from $24.4 \pm$ 2.98 to $74.44 \pm 7.95$. Since 12 months postoperatively, significant differences existed in postoperative HHS between the two groups $(P<0.05)$, and Group PFNA was higher than Group DHS in terms of postoperative HHS.

Duration of surgery was more for DHS compared to PFN. The duration of surgery as calculated from the time of incision to skin closure was counted in each case. The average duration of surgery for the PFN (Avg. time $38 \mathrm{~min}$ ) was significantly shorter then DHS (Avg. time $57 \mathrm{~min}$ ). Blood loss was measured by mop count and collection in suction drain. Blood loss was more for DHS. The average blood loss in the PFN group was $100 \mathrm{ml}$ and in the DHS group was $250 \mathrm{ml} .05$ out of 25 patients in DHS group required blood transfusion either intra or postoperatively. Patients in PFN group had fewer complications as compared to those in DHS group and the difference was statistically significant (Table-1).

Table 1: Complications of DHS and PFN

\begin{tabular}{|c|c|c|}
\hline Complications & DHS Group & PFN Group \\
\hline Pressure sores & 2 & 0 \\
\hline Deep vein thrombosis & 1 & 0 \\
\hline Periprosthetic fracture & 1 & 0 \\
\hline Limb length discrepancy & 4 & 1 \\
\hline Malunion & 2 & 1 \\
\hline Nonunion & 1 & 0 \\
\hline Implant cutout & 2 & 0 \\
\hline Infection & 1 & 0 \\
\hline Re-operation rate & 4 & 0 \\
\hline
\end{tabular}

\section{Discussion}

The debate continues about how best to select implant of intramedullary fixation or extramedullary fixation for stable/unstable IT fractures, and there has not been a conclusive answer in the literature whether stable IT fractures are excellently treated with a Dynamic hip screw(DHS) or a proximal femoral nail( PFN) ${ }^{[12,13]}$. Some randomized clinical trials (RCTs) found no difference of implants in long-term functional outcome between the two devices ${ }^{[3-6]}$. Bhandari et al. ${ }^{[14]}$ reported that there were no large differences in IT fractures risk between the DHS and the PFN. Nevertheless, a meta-analysis reported that a higher reoperation rate occurred after a DHS ${ }^{[13]}$. Jones et al. ${ }^{[15]}$ concluded a DHS should not be recommended for stable IT fractures. Because there were higher fixation failure rates, postoperative femoral fractures in particular after DHS fixation.

In this study, we compared PFN and DHS fixations for the treatment of IT fractures. Our results suggested that PFN device was better than DHS device, as indicated by significantly less total complications, less orthopaedic complications, less post-operative femoral fractures after implant removal, less reoperation rate, and higher 
postoperative HHS. Furthermore, our results highlight the fact that patients with IT fractures may not always need DHS fixation, which, though a satisfactory short-term effect, carries an increased risk of post-operative problems and complications.

Saudan et al. ${ }^{[16]}$ concluded that intramedullary nails (such as the PFN) offer no advantage over extramedullary devices (such as the DHS) for the treatment of intertrochanteric fractures caused by low-energy trauma (AO types 31.A1 and 31.A2). Another study ${ }^{[17]}$ compared functional recovery 1 year after trochanteric nail or DHS repair. Final assessment was conducted over the phone, and no between-group differences in recovery scores were found. In a randomised, prospective study comparing intramedullary versus extramedullary fixation, Baumgaertner et al. ${ }^{[18]}$ found no differences between the groups in functional recovery rates.

Saarenpää et al. ${ }^{[19]}$ compared functional outcomes with the DHS and Gamma Nail fixation systems in terms of parameters including ability to walk and ability to dress and undress both pre-operatively and 4 months after surgery. Although there were no between-group differences in the use of walking aids, in the DHS group, 4-month outcomes were significantly better than pre-injury levels compared with the Gamma Nail group.

In the current study, there was a significant loss of reduction in patients in DHS group and hence more cases of malunion and functional loss (lesser Harris hip score) was reported, whereas no such loss of reduction, function (better Harris hip score) occurred in the PFN-treated group ( $\mathrm{p}=0.097)$, unlike in the Saarenpää et al. study ${ }^{[23]}$. The complication rate in PFN group as compared to other studies previously mentioned (most of them using the Gamma nails), was less as were well versed with the technique and principles of PFN fixation, due to the high volume of IT fracture cases we do at our center. Hence we abided by the principles of PFN fixation in IT fractures, therefore we could achieve good to excellent results. Little et al. ${ }^{[20]}$ also found that intramedullary nailing enabled faster recovery of mobility.

Early stabilisation of unstable intertrochanteric fractures with mechanically more stable implants (such as the PFN) enables earlier weight bearing, recovery of ambulatory function, and return to prefracture social and community function. Intertrochanteric fractures involving the posteromedial wall or lesser trochanter are considered unstable. In patients with such fractures treated with the DHS, weight bearing is delayed until bone union, so as to minimise collapse of the fixation. However, if such fractures are treated with the PFN, early weight bearing is allowed because the PFN is more stable biomechanically. Thus, our PFN patients had better ambulatory independence at 6 and 12 months.

Regarding functional recovery, Dujardin et al. ${ }^{[21]}$ showed that significant differences favouring the intramedullary nail group were apparent 6 months after surgery. This is consistent with the findings in the present study, where functional recovery was faster in PFN treated patients in the first 6 months after surgery. However, the authors found no differences in walking ability or recovery of independence. The finding that functional recovery in the first 6 months after surgery is significantly poorer after DHS treatment is important, because it is widely known that elderly patients require faster recovery. Similar findings have been reported by Goulidakis et al. ${ }^{[22]}$ and Calderón et al. ${ }^{[23]}$. Conversely, Eschler et al. ${ }^{[24]}$ found that patients treated with an extramedullary device fared subjectively better. Ultimately, however, both groups had similar functional recovery outcomes and no significant loss of function at 1-year follow-up.

Taken together, these data indicate that despite similar final scores, the most important result is that patients treated with the PFN technique exhibited functional improvement as early as 6 months after surgery, unlike DHS-treated patients. In practice, this means that PFN treatment is associated with faster improvement in quality of life than the DHS technique. The intramedullary devices offer certain distinct advantages: The implant itself serves as a buttress against lateral translation of the proximal fragment. The intramedullary location of the junction between the nail and lag screw makes the implant stronger at resisting the binding forces. The intramedullary device has a reduced distance between the weight bearing axis and the implant that is a shorter lever arm. An intramedullary device bears the bending load which is transferred to the intramedullary nail and is resisted by its contact against the medullary canal (load sharing device), the intramedullary hip screw is a more biological method of fixation. For the above mentioned reasons it was believed that the intramedullary hip screw would be superior for the fixation of intertrochanteric fractures.

\section{Conclusion}

At 1 year follow-up, unstable IT fractures treated surgically with PFN fared better in terms of functional recovery, functional outcome, and also had fewer orthopaedic and nonorthopaedic complications and hence lesser re-operation rate as compared to those treated with DHS. However PFN as compared to DHS has a slight longer learning curve.

\section{Disclosure}

No external funding taken for the study and there is no conflicts of interest, declared by the authors.

\section{References}

1. Matre K, Havelin LI, Gjertsen JE, Vinje T, Espehaug B, Fevang JM. Sliding hip screw versus IM nail in reverse oblique trochanteric and subtrochanteric fractures. A study of 2716 patients in the Norwegian Hip Fracture Register. Injury. 2013; 44(6):735-42.

2. McCormack R, Panagiotopolous K, Buckley R, Penner M, Perey B, Pate G et al. A multicentre, prospective, randomised comparison of the sliding hip screw with the Medoff sliding screw and side plate for unstable intertrochanteric hip fractures. Injury. 2013; 44(12):19049.

3. Chang JD, Yoo JH, Reddy P, Lee SS, Hwang JH, Kim TY. Risk factors for contralateral hip fracture in elderly patients with previous hip fracture. Injury. 2013; 44(12):1930-3.

4. Kuzyk PR, Lobo J, Whelan D, Zdero R, McKee MD, Schemitsch EH. Biomechanical evaluation of extramedullary versus intramedullary fixation for reverse obliquity intertrochanteric fractures. J Orthop Trauma. 2009; 23:31-8.

5. Wordsworth DR, Halsey $\mathrm{T}$, Griffiths R, Parker MJ. Clopidogrel has no effect on mortality from hip fracture. Injury. 2013; 44(6):743-6.

6. Kadar A, Chechik O, Katz E, Blum I, Meghiddo G, Salai $\mathrm{M}$ et al. The effects of 'old' red blood cell transfusion on mortality and morbidity in elderly patients with hip fractures--a retrospective study. Injury. 2013; 44(6):74750.

7. Koval KJ, Heckman JD. Intertrochanteric fractures. In: Bucholz RW, Heckman JD, editors. Rockwood and 
Green's Fractures in Adults. Philadelphia: Lippincott Williams \& Wilkins, 2001, 1635-63.

8. Hersche O, Heim D, Bodoky A, Regazzoni P. [4 fragment fractures of the proximal femur: is the dynamic hip screw a suitable implant?]. Helv Chir Acta. 1989; 56:577-80.

9. Efstathopoulos NE, Nikolaou VS, Lazarettos JT. Intramedullary fixation of intertrochanteric hip fractures: a comparison of two implant designs. Int Orthop. 2007; 31:71-6.

10. Frohlich P, Benko T. [DHS (Dynamic Hip Screw)osteosynthesis in the management of femoral fractures in the hip region and the place of this method in the treatment of such injuries]. Magy Traumatol Ortop Kezseb Plasztikai Seb. 1993; 36:59-64.

11. Boldin C, Seibert FJ, Fankhauser F, Peicha G, Grechenig W, Szyszkowitz R. The proximal femoral nail (PFN)--a minimal invasive treatment of unstable proximal femoral fractures: a prospective study of 55 patients with a follow-up of 15 months. Acta Orthop Scand. 2003; 74:53-8.

12. Zhang K, Zhang S, Yang J, Dong W, Wang S, Cheng Y et al. Proximal femoral nail vs. dynamic hip screw in treatment of intertrochanteric fractures: a meta-analysis. Med Sci Monitor. 2014; 20:1628-33.

13. Ma KL, Wang X, Luan FJ, Xu HT, Fang Y, Min J et al. Proximal femoral nails antirotation, Gamma nails, and dynamic hip screws for fixation of intertrochanteric fractures of femur: a meta-analysis. Orthop Traumatol Surg Res. 2014; 100(8):859-66.

14. Bhandari M, Schemitsch E, Joensson A, Zlowodzki M, Haidukewych GJ. Gamma nails revisited: gamma nails versus compression hip screws in the management of intertrochanteric fractures of the hip: a meta-analysis. J Orthop Trauma. 2009; 23(6):460-4.

15. Jones HW, Johnston P, Parker M. Are short femoral nails superior to the sliding hip screw? a meta-analysis of 24 studies involving 3,279 fractures. Int Orthop. 2006; 30(2):69-78.

16. Saudan M, Lubbeke A, Sadowski C, Riand N, Stern R, Hoffmeyer P. Pertrochanteric fractures: is there an advantage to an intramedullary nail?: a randomized, prospective study of 206 patients comparing the dynamic hip screw and proximal femoral nail. J Orthop Trauma. 2002; 16:386-93.

17. Crawford $\mathrm{CH}$, Malkani AL, Cordray S, Roberts CS, Sligar W. The trochanteric nail versus the sliding hip screw for intertrochanteric hip fractures: a review of 93 cases. J Trauma. 2006; 60:325-8; discussion 328-9.

18. Baumgaertner MR, Curtin SL, Lindskog DM. Intramedullary versus extramedullary fixation for the treatment of intertrochanteric hip fractures. Clin Orthop Relat Res. 1998; 348:87-94.

19. Saarenpaa I, Heikkinen T, Ristiniemi J, Hyvonen P, Leppilahti J, Jalovaara P. Functional comparison of the dynamic hip screw and the Gamma locking nail in trochanteric hip fractures: a matched-pair study of 268 patients. Int Orthop. 2009; 33:255-60.

20. Little NJ, Verma V, Fernando C, Elliott DS, Khaleel A. A prospective trial comparing the Holland nail with the dynamic hip screw in the treatment of intertrochanteric fractures of the hip. J Bone Joint Surg Br. 2008; 90:10738.

21. Dujardin FH, Benez C, Polle G, Alain J, Biga N, Thomine JM. Prospective randomized comparison between a dynamic hip screw and a mini-invasive static nail in fractures of the trochanteric area: preliminary results. J Orthop Trauma. 2001; 15:401-6.

22. Goulidakis V, Theocharakis S, Diakatos A, Gourovanidis D, Drakoulakis E, Pastroudis A. A35 Comparative study of trochanteric fractures treated with the Gamma nail-G3 and Dynamic Hip Screw. Results and clinical outcome. Injury. 2011; 42:S9.

23. Calderon A, Ramos T, Vilchez F, Mendoza-Lemus O, Pena V, Cardenas-Estrada E et al. [Proximal femoral intramedullary nail versus DHS plate for the treatment of intertrochanteric fractures. A prospective analysis]. Acta Ortop Mex. 2013; 27:236-9.

24. Eschler A, Brandt S, Gierer P, Mittlmeier T, Gradl G. Angular stable multiple screw fixation (Targon $\mathrm{FN}$ ) versus standard SHS for the fixation of femoral neck fractures. Injury. 2014; 45:S76-80. 